\title{
VANISHING THEOREMS FOR SINGULAR VARIETIES
}

\author{
KYUNGHO OH
}

(Communicated by Eric M. Friedlander)

\begin{abstract}
We give generalizations of Ramanujam's version of the Kodaira Vanishing Theorem to singular varieties.
\end{abstract}

In this note, we give generalizations of the Kodaira Vanishing Theorem to singular varieties. Let $X$ be an $n$-dimensional Cohen-Macaulay projective variety over $\mathbb{C}$ and $L$ be a line bundle on $X$. For $s \in V=H^{0}\left(X, L^{m}\right), s \neq 0$, let $D_{s}$ be the zero-scheme of $s$, a Cartier divisor on $X$. Let $B=\bigcap_{s \in V-\{0\}} D_{s}$ be the base locus of the linear system $V$. Then, there is a rational map $\phi_{L^{m}}: X \rightarrow \mathbb{P}\left(V^{*}\right)$ defined outside $B$ by sending $x$ to the hyperplane of divisors containing $x$.

Theorem 1. Let $X, L$, and $\phi_{L^{m}}$ be as above and $B$ be the base locus of the linear system $H^{0}\left(X, L^{m}\right)$. Suppose that the dimension of the image of $X$ under the map $\phi_{L^{m}}$ is at least $k$. Then

$$
H^{i}\left(X, \omega_{X} \otimes L\right)=0 \text { for } i>\max (n-k, \operatorname{dim} B, \operatorname{dim} \operatorname{Sing}(X)),
$$

where $\operatorname{Sing}(X)$ denotes the singular locus of $X$.

Remark 1 . When $X$ is a nonsingular projective variety over $\mathbb{C}$, this was proved by Ramanujam [R, Theorem 3].

Proof. First of all, we reduce the case to $m=1$ using a finite covering of $X$. By Bertini's Theorem, there is a basis $\left\{s_{0}, \ldots, s_{r}\right\}$ of the complete linear system $H^{0}\left(X, L^{m}\right)$ such that the zero-scheme $D_{s_{i}}$ of $s_{i}$ is smooth outside the union of $B$ and $\operatorname{Sing}(X)$ for $i=0, \ldots, r$. Now we construct a tower of cyclic coverings inductively. We set $Y_{-1}=X$ and $\tau_{-1}$ to be the identity map on $X$. The section $\tau_{j-1}^{*}\left(s_{j}\right)$ of $\tau_{j-1}^{*}\left(L^{m}\right)$ defines a structure of an $\mathscr{O}_{Y_{j-1}}$-algebra on $\bigoplus_{i=0}^{m-1} \tau_{j-1}^{*} L^{-i}$. The natural map

$$
\sigma_{j}: Y_{j}=\operatorname{Spec}_{Y_{j-1}}\left(\bigoplus_{i=0}^{m-1} \tau_{j-1}^{*} L^{-i}\right) \rightarrow Y_{j-1}
$$

Received by the editors January 13, 1992.

1991 Mathematics Subject Classification. Primary 14F17, 32L20.

(C) 1993 American Mathematical Society $0002-9939 / 93 \$ 1.00+\$ .25$ per page 
gives an $m$-fold cyclic covering map of $Y_{j-1}$. Let

$$
\tau_{j}=\sigma_{j} \circ \tau_{j-1}: Y_{j} \rightarrow X
$$

be the composition map and $\tau=\tau_{r}$. Then $Y_{r}$ will be smooth outside the inverse image $\tau^{-1}(B \cup \operatorname{Sing}(X))$. In particular, the maximum of the dimensions of $B$ and $\operatorname{Sing}(X)$ will be preserved under the map $\tau$. Also note that $\tau^{*} L$ possesses sections $t_{0}, \ldots, t_{r}$ such that $t_{i}^{m}=\tau^{*}\left(s_{i}\right)$ in $\tau^{*} L^{m}$. Thus the field $\mathbb{C}\left(t_{1} / t_{0}, \ldots, t_{r} / t_{0}\right)$ is algebraic over the function field $\mathbb{C}\left(s_{1} / s_{0}, \ldots, s_{r} / s_{0}\right)$ of $\phi_{L^{m}}(X)$. Hence the dimension of the image of $Y_{r}$ under the map $\phi_{\tau^{*} L}$ is at least $k$ since the function field $K\left(\phi_{\tau^{*} L}\left(Y_{r}\right)\right)$ contains $\mathbb{C}\left(t_{1} / t_{0}, \ldots, t_{r} / t_{0}\right)$. Furthermore, we have

$$
H^{i}\left(X, \tau_{*} \tau^{*} L^{-1}\right)=H^{i}\left(Y_{r}, \tau^{*} L^{-1}\right),
$$

since $\tau$ is affine. The trace map gives a splitting of the natural homomorphism $L^{-1} \rightarrow \tau_{*} \tau^{*} L^{-1}$, and hence $L^{-1}$ is a direct summand of $\tau_{*} \tau^{*} L^{-1}$. Moreover, $Y_{r}$ is Cohen-Macaulay since $\tau$ is flat. Now by Serre duality, $H^{i}\left(Y_{r}, \omega_{Y_{r}} \otimes \tau^{*} L\right)=0$ will imply that $H^{i}\left(X, \omega_{X} \otimes L\right)=0$. Hence we may assume $m=1$.

Let $B$ be the base locus of the linear system $V=H^{0}(X, L)$. Let $\pi: \widetilde{X} \rightarrow X$ be a desingularization of the blow-up of $X$ along $B$. There is a morphism

$$
\psi: \widetilde{X} \rightarrow \mathbb{P}\left(V^{*}\right)
$$

extending the morphism $\phi_{L}: X-B \rightarrow \mathbb{P}\left(V^{*}\right)$. Then we have

$$
\psi^{*} \mathscr{O}(1)=\pi^{*} L \otimes \mathscr{O}(-E)
$$

where $E$ is an effective divisor on $\widetilde{X}$ with support in $\pi^{-1}(B)$. Thus there is an exact sequence

$$
0 \rightarrow \psi^{*} \mathscr{O}(1) \otimes \omega_{\tilde{X}} \rightarrow \pi^{*} L \otimes \omega_{\tilde{X}} \rightarrow \pi^{*} L \otimes \omega_{\tilde{X}} \otimes \mathscr{O}_{E} \rightarrow 0 .
$$

We claim that

$$
R^{q} \pi_{*}\left(\psi^{*} \mathscr{O}(1) \otimes \omega_{\tilde{X}}\right)=0 \quad \text { for } q>0 .
$$

Let $H$ be a smooth divisor of a section of $\psi^{*} \mathscr{O}(1)$. Consider the Poincaré residue sequence

$$
0 \rightarrow \omega_{\tilde{X}} \rightarrow \mathscr{O}(H) \otimes \omega_{\tilde{X}} \rightarrow \omega_{H} \rightarrow 0 .
$$

Grauert-Riemenschneider Vanishing Theorem [GR, Satz 2.3] implies that

$$
R^{q} \pi_{*} \omega_{\tilde{X}}=R^{q} \pi_{*} \omega_{H}=0 \text { for } q>0 .
$$

Thus we conclude that

$$
R^{q} \pi_{*}\left(\psi^{*} \mathscr{O}(1) \otimes \omega_{\tilde{X}}\right)=R^{q} \pi_{*}\left(\mathscr{O}(H) \otimes \omega_{\tilde{X}}\right)=0 \quad \text { for } q>0 .
$$

Hence we get from (1)

$$
0 \rightarrow \pi_{*}\left(\psi^{*} \mathscr{O}(1) \otimes \omega_{\tilde{X}}\right) \rightarrow \pi_{*}\left(\pi^{*} L \otimes \omega_{\tilde{X}}\right) \rightarrow \pi_{*}\left(\pi^{*} L \otimes \omega_{\tilde{X}} \otimes \mathscr{O}_{E}\right) \rightarrow 0 .
$$

Since $\pi_{*}\left(\pi^{*} L \otimes \omega_{\tilde{X}} \otimes \mathscr{O}_{E}\right)$ is supported on $B$, there is a surjection

$$
H^{i}\left(X, \pi_{*}\left(\psi^{*} \mathscr{O}(1) \otimes \omega_{\tilde{X}}\right)\right) \rightarrow H^{i}\left(X, \pi_{*}\left(\pi^{*} L \otimes \omega_{\tilde{X}}\right)\right) \text { for } i>\operatorname{dim} B .
$$


(In fact, this is an isomorphism for $i>\operatorname{dim} B+1$. ) Via the Leray spectral sequence, this induces a surjection

$$
H^{i}\left(\tilde{X}, \psi^{*} \mathscr{O}(1) \otimes \omega_{\tilde{X}}\right) \rightarrow H^{i}\left(\tilde{X}, \pi^{*} L \otimes \omega_{\tilde{X}}\right) \text { for } i>\operatorname{dim} B
$$

since $R^{q} \pi_{*}\left(\pi^{*} L \otimes \omega_{\tilde{X}}\right)=L \otimes R^{q} \pi_{*} \omega_{\tilde{X}}=0, q>0$, by the Grauert-Riemenschneider Vanishing Theorem [GR, Satz 2.3].

On the other hand, we have

$$
H^{i}\left(\tilde{X}, \psi^{*} \mathscr{O}(1) \otimes \omega_{\tilde{X}}\right)=0 \text { for } i>n-k,
$$

by Ramanujam's generalization of the Kodaira Vanishing Theorem [R, Theorem 3]. Therefore, we have

$$
H^{i}\left(\tilde{X}, \pi^{*} L \otimes \omega_{\tilde{X}}\right)=0 \text { for } i>\max (n-k, \operatorname{dim} B) .
$$

Again from the Leray spectral sequence and the Grauert-Riemenschneider Vanishing Theorem [GR, Satz 2.3], it follows that

$$
H^{i}\left(X, L \otimes \pi_{*} \omega_{\tilde{X}}\right)=0 \quad \text { for } i>\max (n-k, \operatorname{dim} B) .
$$

Now our proof follows from an exact sequence

$$
0 \rightarrow \pi_{*} \omega_{\widetilde{X}} \rightarrow \omega_{X} \rightarrow Q \rightarrow 0
$$

where $Q$ is supported on $\operatorname{Sing}(X)$.

Corollary 2. Let $X$ be an $n$-dimensional projective variety over $\mathbb{C}$ with only rational singularities outside the base locus $B$ of $H^{0}\left(X, L^{m}\right)$. Suppose that the dimension of the image of $X$ under the map $\phi_{L^{m}}$ is at least $k$. Then

$$
H^{i}\left(X, \omega_{X} \otimes L\right)=0 \text { for } i>\max (n-k, \operatorname{dim} B) .
$$

Proof. First, we will show that the reduction step to $m=1$ works. Let $\pi: \widetilde{X} \rightarrow$ $X$ be a desingularization of the blow-up of $X$ along $B$. By Bertini's Theorem, there is a basis $\left\{s_{0}, \ldots, s_{r}\right\}$ of $H^{0}\left(X, L^{m}\right)$ such that the zero schemes of $\pi^{*}\left(s_{i}\right)$ are of the form $H_{i}+E$ where $H_{i}$ are smooth hypersurfaces and $E$ is supported on $\pi^{-1}(B)$. Let $\tau: Y_{r} \rightarrow X$ be a branched covering of $X$ over the union of the divisors $D_{s_{i}}$ constructed as in the proof of the theorem. Consider the fibre product:

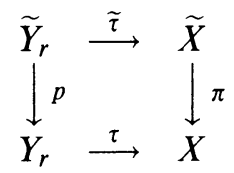

Since cohomology commutes with flat base extension [H, III, 9.3], there are natural isomorphisms

$$
R^{i} p_{*} \mathscr{O}_{\widetilde{Y}_{r}} \cong \tau^{*} R^{i} \pi_{*} \mathscr{O}_{\widetilde{X}}
$$

Since $X$ has only rational singularities outside $B$, we have that $R^{i} p_{*} \mathscr{O}_{\widetilde{Y}_{r}}$ is supported on $\tau^{-1}(B)$ when $i>0$ and $p_{*} \mathscr{O}_{\widetilde{Y}_{r}}=\mathscr{O}_{Y_{r}}$ on $Y_{r}-\tau^{-1}(B)$. Moreover, $\tilde{Y}_{r}$ is smooth outside $\tilde{\tau}^{-1}(E)$ since the zero schemes of $\pi^{*}\left(s_{i}\right)$ are smooth on $X-E$. Thus $Y_{r}$ has only rational singularities outside $\tau^{-1}(B)$. Note that the dimension of $B$ is unchanged under the map $\tau$. Hence we may assume $m=1$. 
From [K, Proposition, p. 50], we obtain an exact sequence

$$
0 \rightarrow \pi_{*} \omega_{\tilde{X}} \rightarrow \omega_{X} \rightarrow Q \rightarrow 0
$$

where $Q$ is supported on the base locus $B$. The corollary follows from (4) and this sequence.

\section{ACKNOWLEDGMENT}

I am grateful to Professor Donu Arapura for various suggestions and help. This work started during my stay at Purdue University in the summer of 1991. I would like to thank the Department of Mathematics of Purdue University for its hospitality.

\section{REFERENCES}

[GR] H. Grauert, and O. Riemenschneider, Verschwindungssätze für analytische Kohomologiergruppen auf komplexen Räumen, Invent. Math. 11 (1970), 263-292.

[H] R. Hartshorne, Algebraic geometry, Springer-Verlag, New York, 1970.

[K] G. Kempf, Toroidal embeddings 1, Lecture Notes in Math., vol. 339, Springer-Verlag, New York, 1973.

[R] C. P. Ramanujam, Remarks on the Kodaira vanishing theorem, J. Indian Math. Soc. 36 (1972), 41-50.

Department of Mathematics and Computer Science, University of Missouri-St. Louis, ST. Louis, Missouri 63121

E-mail address: oh@arch.umsl.edu 